\title{
Von der Bedarfserhebung zur Uraufführung II
}

\author{
Über Herstellung und Vertrieb audiovisueller Medien für religiöse Erziehung \\ und kirchliche Bildungsarbeit
}

von Anton Täubl

\begin{abstract}
In diesem Heft findet der Beitrag Anton Täubls über Probleme der Herstellung und des Vertriebs einer Dia-Serie bzw. eines Kurzfilms seine abschließende Fortsetzung. Beschäftigte sich der erste Teil des Beitrages in CS 3/1984 vorwiegend mit der Planung und Finanzierung von Kurzfilmen religiöser Inhalte, so wendet sich der Autor in diesem zweiten Teil dem Problemkreis der Dreharbeiten, der Werbung sowie dem Vertrieb und dem Verkauf zu.
\end{abstract}

\subsubsection{Durchführung der Produktion - Dreharbeiten und Abnahmen}

Aufnahmen beginnen nach Abnahme des Drehbuchs und nach Abschluß des Produktionsvertrags. Sie laufen in verschiedenen Phasen ab:

a) Drehvorbereitungen: Produktions- und Aufnahmeleiter tragen hierfür die entscheidende Verantwortung. Sie sorgen für den reibungslosen organisatorischen (und menschlichen) Ablauf der Dreharbeiten. Zwei Eigenschaften sollten sie auszeichnen: Zum einen müssen sie organisieren können, oft auch improvisieren; zum anderen den Kontakt zu allen Mitarbeitern halten und die Atmosphäre entspannen, wenn dunkle Wolken aufziehen und Irritationen sich breit machen.

b) Aufnahmearbciten: Der Film entsteht nach einem Drehplan, der unter wirtschaftlichen und kreativen Gesichtspunkten erstellt wird. Zum Regie- und Aufnahmestab für einen Kurzspielfilm gehören folgende Personen, die jeweils besondere Funktionen ausüben: Regisseur, Regieassistent, Produktionsleiter, Aufnahmeleiter, Kameramann, Kamera-Assistent, Requisiteur, Maskenbildner, Tonmeister, Tonassistent, Oberbeleuchter, Beleuchter, Betreuer für Kinder und Jugendliche (falls diese mitwirken), Darsteller. Wieviel an einem Tag abgedreht werden kann, ist - entsprechend den Filmgattungen - jeweils unterschiedlich. Dokumentarfilme sind an Ereignisse und ihren Ablauf gebunden; bei nachgestellten Dokumentationen und vor allem bei Spielfilmen können Einstellungen wiederholt werden, zum Beispiel im Verhältnis 1 zu 8 oder 1 zu 15, das heißt: Eine Szene, die später im Film zu sehen ist, wird bis zu 8 oder 15 mal aufgenommen. Die Einstellung ist die kleinste Einheit, die bei Filmaufnahmen ohne Unterbrechung abgedreht wird. Pro Tag entstehen im Schnitt 7wischen drei bis acht Minuten verwertbare Filmlaufzeit.

Abgedrehte Filme werden ins Kopierwerk gebracht, entwickelt und vom Regisseur (und Kameramann) meist schon am selben oder am darauffolgenden Tag begutachtet: Es wird festgestellt, ob dic Aufnahmen technisch und darstellerisch zufriedenstellend ausgefallen sind.

Mcinungsverschiedenheiten und Konflikte, die bei Dreharbeiten immer wieder entstehen können, werden in der Regel mit dem Aufnahmeleiter oder Produktionsleiter geregelt; in

Anton Täubl ist Produktionsreferent für Religion und Ethik am „Institut für Film und Bild in Wissenschaft und Unterricht", FWU. Er ist auch Mitglied der Arbeitsgruppe audiovisuelle Medien der Zentralstelle Medien der Deutschen Bischofskonferenz. 
schweren Fällen wird der Hersteller (Realisator) eingeschaltet und unter Umständen auch der Redakteur beziehungsweise der Projektbetreuer von seiten der auftraggebenden Institution.

c) Rohschnitt, Feinschnitt, Endabnahme: Nach der Entwicklung des Filmmaterials werden vom Cutter (Schnittmeister) am Schneidetisch die Einstellungen in der richtigen Folge aneinandergereiht. Zunächst bilden einzelne Szenen größere Komplexe, den sogenannten Grobschnitt (Rohschnitt). Regisseur und Cutter erörtern am vorliegenden Material Fragen der Endfertigung. Beim Feinschnitt kommt der Film seiner endgültigen Gestalt bereits sehr nahe. Ab diesem Zeitpunkt wird nur noch über Details der Endfassung diskutiert - obwohl auch jetzt noch substanzielle Umstellungen oder Veränderungen möglich wären, falls diese wider Erwarten erwogen werden sollten.

Nach dem Feinschnitt werden Bild und Ton, die durchgehend getrennt aufgenommen und separat verarbeitet worden sind, zum Tonfilm zusammengefügt; soweit erforderlich, wird jetzt auch der Kommentar in seiner end gültigen Fassung, ,angelegt “. So entsteht die O-Kopie (Null-Kopie), die Endfassung des Films. Bei der Endabnahme wird anhand einer O-Kopie, meist mit Magnettonrandspur versehen (kombinierte Kopie mit Bild und Ton), eine letzte technische und inhaltliche Überprüfung der Filmfassung durchgeführt, bevor er zur Herstellung von Massenkopien in Auftrag geht.

Nach Abgabe des vom Realisator abzuliefernden Materials beziehungsweise dem entsprechenden Einlagerungsbescheid der Kopieranstalt gilt die Produktion als fertiggestellt; die Schlußrate kann ausbezahlt werden.

d) Kopierausgan gsmaterial und $\mathrm{M}$ assenkopien: Nicht jedes Aufnahmematerial eignet sich in gleicher Weise zur Herstellung von Massenkopien. Für Neuproduktionen eignet sich derzeit folgendes Material:

- Auf 35-mm-Color-Negativfilm: Die besten Ergebnisse sind mit Eastnamcolor II Typ 5247 zu erzielen (vielfältige Zwischenkopiermaterialien, Eignung für Auslieferung in unterschiedlichen Filmformaten sowie Video-Formaten, gute Trickkopier-Möglichkeiten);

- Auf 16-mm-Color-Umkehrfilm: Gute Ergebnisse können mit Ektachrome-Commercial Typ 7252 erzielt werden. Die Eigenheiten des Materials, insbesondere sein geringer Belichtungsspielraum, setzen jedoch langjährige Erfahrungen in der Aufnahmetechnik voraus;

- Auf 16-mm-Color-Negativfilm: Für Produktionen unter ungünstigen Lichtverhältnissen kann der neue Eastnamcolor II Typ 7247 verwendet werden. Die geringere Schärfenleistung dieses Materials schließt unter anderem seine Verwendung für die Herstellung von 8-mm-Filmkopien aus;

- Auf Schwarz-weiß-Materialien: Es ist die Verwendung von Negativ- und Umkehrfilm im 35- und 16-mm-Format möglich. Erforderlich sind feinkörnige Materialien (etwa KodakPlus-X bzw. Plux-X-Reversal oder ähnliche Emulsionen anderer Fabrikate). 
Tabelle: Aufnahmematerialien für Neuproduktionen (Quelle: FWU-Handbuch 111.1)

Beim Ausgangsmaterial für Neuproduktionen ergibt sich folgendes Bild:

\begin{tabular}{|c|c|c|c|c|c|}
\hline $\begin{array}{l}\text { Massen- } \\
\text { produktion } \\
\text { als }\end{array}$ & $\begin{array}{l}\text { Bildinhalt } \\
\text { Filmform }\end{array}$ & $\begin{array}{l}35 \mathrm{~mm} \\
\text { Color- } \\
\text { Negativ }\end{array}$ & $\begin{array}{l}16 \mathrm{~mm} \\
\text { Color- } \\
\text { Negativ }\end{array}$ & $\begin{array}{l}16 \mathrm{~mm} \\
\text { Color- } \\
\text { Umkehr }\end{array}$ & $\begin{array}{c}35 / 16 \mathrm{~mm} \\
\text { Schwarz- } \\
\text { Weiß }\end{array}$ \\
\hline \multirow{2}{*}{$\begin{array}{l}16 \mathrm{~mm}- \\
\text { Film }\end{array}$} & $\begin{array}{l}\text { Hohe Informations- } \\
\text { dichte, z.B. } \\
\text { Totalen } \\
\text { detaillierte Grafiken } \\
\text { Schriftfelder } \\
\text { Titel auf Realuntergrund }\end{array}$ & ja & nein & $\mathrm{ja}^{*}$ & ja \\
\hline & $\begin{array}{l}\text { Geringe Informations- } \\
\text { dichte, z.B. } \\
\text { Großaufnahmen } \\
\text { Halbtotalen } \\
\text { kontrastreiche/einfache } \\
\text { grafische Darstellungen }\end{array}$ & $\mathrm{ja}$ & ja & ja & ja \\
\hline $\begin{array}{c}8 \mathrm{~mm}- \\
\text { Film }\end{array}$ & $\begin{array}{l}\text { Geringe Information- } \\
\text { dichte, z.B. } \\
\text { Großaufnahmen } \\
\text { Halbtotalen } \\
\text { kontrastreiche/einfache } \\
\text { grafische Darstellungen }\end{array}$ & ja & nein & $\mathrm{ja}^{*}$ & $\mathrm{ja}$ \\
\hline Video & $\begin{array}{l}\text { Geringe Informations- } \\
\text { dichte, z.B. } \\
\text { Großaufnahmen } \\
\text { Halbtotalen } \\
\text { kontrastreiche/einfache } \\
\text { grafische Darstellungen }\end{array}$ & $\mathrm{ja}$ & ja & ja & ja \\
\hline
\end{tabular}

*Falls die Aufnahmebedingungen es ermöglichen, ist $35 \mathrm{~mm}$-Film zu verwenden. 


\begin{tabular}{|c|c|c|c|c|c|}
\hline \multirow[b]{2}{*}{ Art/Format } & \multirow{2}{*}{\multicolumn{2}{|c|}{ Bezeichnung }} & \multicolumn{3}{|c|}{ Massenproduktion } \\
\hline & & & $16 \mathrm{~mm}$ & $8 \mathrm{~mm}$ & VCR \\
\hline \multirow{4}{*}{$\begin{array}{c}35 \mathrm{~mm} \\
\text { Farbe }\end{array}$} & 2.11 & Eastmancolor-Negativ Typ 5247 & ja & ja & ja \\
\hline & 2.21 & Gevacolor-Negativ Typ 680 & ja & ja & ja \\
\hline & 2.31 & Intermediate Positiv Typ 5253 & ja & ja & ja \\
\hline & 2.11 & (2 Stuick vom Originalnegativ gezogen) & & & \\
\hline \multirow{3}{*}{$\begin{array}{c}35 \mathrm{~mm} \\
\text { Farbe }\end{array}$} & 2.41 & Originalnegativ & ja & ja & ja \\
\hline & 2.51 & Feinkornpositiv (Lavendel) & ja & ja & ja \\
\hline & 2.51 & (2 Stück vom Originalnegativ gezogen) & & & \\
\hline \multirow{6}{*}{$\begin{array}{l}16 \mathrm{~mm} \\
\text { Farbe }\end{array}$} & 2.61 & Ektachrome-Commercial Typ 7252 & ja & ja & ja \\
\hline & 2.71 & Internegativ Typ 7271 & ja & ja & ja \\
\hline & $2.1 \mathrm{I}$ & (4 Stück vom Original ECO gezogen) & & & \\
\hline & 2.81 & Eastmancolor-Negativ ECN II 7247 & evtl. & nein & ja \\
\hline & 2.91 & CRI-Negativ Typ 7249 & evtl. & nein & ja \\
\hline & 2.21 & (4 Stück vom Original-Negativ gezogen) & & & \\
\hline \multirow[t]{2}{*}{$16 \mathrm{~mm} \mathrm{~S} / \mathrm{W}$} & 2.10 & Umkehroriginal (mit Randnummern) & ja & ja & ja \\
\hline & 2.11 & Ausnahmsweise: Negativ-Material & evtl. & nein & ja \\
\hline \multirow{4}{*}{ Ton } & 3.11 & Mischband (Perfo-Magnetband) & ja & ja & ja \\
\hline & 2.11 & ( 17,5 oder 35 oder $16 \mathrm{~mm}$ Mittenspur) & & & \\
\hline & 3.21 & Internationales Tonband (IT-Band) & ja & ja & ja \\
\hline & 3.11 & (I7.5 oder 35 oder $16 \mathrm{~mm}$ Mittenspur) & & & \\
\hline
\end{tabular}

e) Begleitmaterialien: Für Erziehung, Unterricht und Bildung sollten normalerweise keine AV-Medien, auch keine Filme ausgeliefert werden ohne pädagogisches Begleitmaterial. Das Medienbegleitmaterial richtet sich an den Benutzer der Produktion (Lehrer, Erzieher, Bildungsreferenten, Geistliche usw.), es gibt Hinweise zur Verwendung in der Bildungssituation; es kann auch die im Medium enthaltenen Informationen ergänzen und zur Vertiefung in den jeweiligen Problemkreis anregen. Das Begleitmaterial hat zum Ziel, die Eingliederung des Mediums in den Bildungsablauf zu erleichtern. Als zweckmäßig haben sich folgende Angaben herausgestellt:

- Titel/Untertitel

- Technische Daten wie zum Beispiel Bildzahl oder Laufzeit

- Zielgruppen/Adressaten-Angaben

- Lernziel-Angaben (Intentionen)

- Hinweise auf Vorkenntnisse

- Annotation (kurze Inhaltsangabe, zum Beispiel für Medienlisten und Kataloge) 
- Ausführliche Inhaltsangabe

- Hinweise zur Verwendung, unter Beachtung didaktischer und methodischer Gesichtspunkte

- Zusatzinformationen zur Vertiefung der Thematik

- Hinweise auf weitere AV-Medien und auf Literatur

Medienbegleitkarten dürfen natürlich nicht umfangreich sein, höchstens zwei bis vier Seiten lang. Neben Begleitkarten legt es sich manchmal aus pädagogischen Gründen nahe, jeder Kopie auch „Kopiervorlagen“ beizulegen, mit dem Recht auf Vervielfältigung für einen Gebrauch durch das Publikum; der Dokumentarfilm „Die Sterne zwingen nicht. Astrologie - Schicksalsglaube oder Lebenshilfe" (32 3362) enthält auf einer Kopiervorlage beispielsweise ein Geburtshoroskop mit der Erklärung von Tierkreiszeichen und Planetensymbolen. Manche Themen erfordern a uch umfangreichere Begleithefte, so etwa Filme für den Sprachunterricht oder auch Diareihen, wie beispielsweise eine Produktion über Holzschnitte des Künstlers Walter Habdank „Abraham und sein Sohn/Abraham und sein Land“ (102510/10 2511) mit grundsätzlichen exegetischen, katechetischen und kunsterzieherischen Angaben zur Patriarchengestalt des Abraham und zur Arbeit des Künstlers.

\subsubsection{Produktionsbegleitende Maßnahmen}

Die Produzenten-beziehungsweise Auftraggeberseite wird durch den Redakteur oder Projektbetreuer vertreten. Seine Aufgabe ist es, den Produktionsablauf bis zur Fertigstellung des Mediums zu begleiten. Er ist Gesprächspartner für die Auftragnehmerseite. Auftauchende Probleme werden mit ihm besprochen und einer Lösung zugeführt; nur in wichtigen Fragen oder in kritischen Situationen werden auch noch andere Instanzen eingeschaltet. Ein Verhältnis mit dem Aufnahmeteam, das von Vertrauen und Sachkenntnis getragen wird, erweist sich als sehr vorteilhaft. $\mathrm{Zu}$ empfehlen ist auch, daß der Redakteur bald nach Drehbeginn am Aufnahmeort erscheint und sich ein Bild über den Fortlauf der Arbeiten und die Atmosphäre innerhalb des Teams macht; es legt sich nahe, den „Besuch“ zu wiederholen. Es obliegt dem Redakteur, die Rohschnittfassung zu diskutieren, den Feinschnitt abzunehmen und auch die Endabnahme zu begutachten - falls dies nicht durch ein Gremium des Auftraggebers geschieht. Jede Produktion, die zur Herstellung vergeben wird, benötigt einen Projektbetreuer, den Redakteur.

\subsubsection{Risiken der Produktion}

In der Medienherstellung - hier am Beispiel der Filmproduktion aufgezeigt - lassen sich vorwiegend folgende Risikoquellen feststellen:

a) Mit Unsicherheitsfaktoren belastet sind Themenwahl und Zielgruppenbestimmung. Es gibt AV-Medien, auch Filme, die am Bedarf vorbei produziert werden. In dem Bereich läßt sich das Risiko um so mehr mindern, je sorgfältiger Fragen der Bedarfserhebung und Bedarfsplanung beachtet werden (vgl. I).

b) Risiken ergeben sich aber auch aus der technischen und künstlerischen Eigenart der Medienproduktion, wozu beispielsweise individuelle Leistungen des Drehbuchautors, Regisseurs, der Darsteller, des Kameramanns, Tonmeisters, Schnittmeisters und so weiter gehören. Ein guter Redakteur, die Wahl qualifizierten Fachpersonals und eine normal gute finanzielle Ausstattung tragen zur Begrenzung minderwertiger Produktionen erheblich bei.

c) Schließlich gibt es noch Unsicherheitsfaktoren, die mit Vertrieb und Absatz der Produktionen zusammenhängen: In einer Phase wirtschaftlicher Rezession geht der Verkauf von 
AV-Medien zurück; auch unvorhersehbare pädagogisch-bildungspolitische Trends, die sich negativ auf die Medienverwendung auswirken - etwa eine derzeit zunehmende Medienmüdigkeit - bilden Risikofaktoren für die kommerzielle Auswertung der Produktionen.

\section{Werbung - Vertrieb - Verkauf}

Werbe-, Vertriebs- und Verkaufsmaßnahmen sollen über neue AV-Medien informieren, zum Kauf derselben reizen, ihren Absatz steigern, sie verbreiten, um damit letzten Endes die Seelsorgs- und Bildungsbemühungen attraktiver und effektiver zu gestalten.

a) Werbemaßnahmen dienen dazu, eine möglichst breite Öffentlichkeit über AV-Medien zu informieren. Entscheidend dabei ist, daß die richtigen Zielgruppen angesprochen, daß Meinungsbildner und Entscheidungsträger erreicht werden. $\mathrm{Zu}$ beachten sind folgende Fragenkreise:

- was will die Werbemaßnahme erreichen (Absichten, Ziele)?

- wer soll angesprochen werden (Adressaten)?

- wie geht man am wirksamsten vor (Verfahren, Methoden)?

- wie geht man am wirtschaftlichsten vor (ökonomische Aspekte)?

Die Werbung kündigt Produkte an, weist auf sie hin, macht sie bekannt und wirbt um das Vertrauen potentieller Kunden. Es sollte stets darauf geachtet werden, daß die Firma (AVVerlag, Produzent) über alle unterschiedlichen Werbemaßnahmen hinaus immer als solche identifiziert werden kann; dies erfolgt über das Firmenzeichen: das Signet! Als besonders wirksam erweist sich die zielgruppenorientierte Werbung, im Gegensatz zur Streuwerbung. Werbemittel und Werbewege sind unter anderem: Verkaufsangebote, Werbeprospekte aller Art, Fachkataloge, Gesamtkataloge, Anzeigenwerbung sowie Hinweise und Rezensionen in Zeitungen und Fachzeitschriften, Bereitstellung von Ansichtskopien, Sichtveranstaltungen, Vorstellung neuer Produktionen auf Ausstellungen und Messen sowie bei Medienbörsen und Filmfestivals, persönliche Kontakte und Besuche (die am wichtigsten sind). Informative Fachmessen von AV-Medien sind in der Bundesrepublik Deutschland vor allem für kirchlich-orientierte Produzenten:

- „Kleinmedien börse Bild/Ton“, veranstaltet vom Gemeinschaftswerk der evangelischen Publizistik e.V. in Zusammenarbeit mit der Arbeitsgemeinschaft der evangelischen AVMedienzentralen unter Beteiligung von Interessenten und Institutionen aus dem katholischen Bereich. Ort: Wechselnd; Zeit: Jeweils im Frühjahr.

- „Tage des Internationalen religiösen Films“ für A mateure und Professionelle, eine private Initiative, veranstaltet vom evangelischen Pastoren-Ehepaar Karen und Dietmar Ennulat, unter Beteiligung örtlicher Organisationen und kommunaler Einrichtungen. Ort: Friedberg bei Frankfurt am Main/Hessen; Zeit: Spätherbst.

- „Medienbörse Film“, eine ökumenische Veranstaltung, die zunehmend an Bedeutung gewinnt, verantwortlich getragen vom Fachbereich Film-Bild-Ton im Gemeinschaftswerk der evangelischen Publizistik e.V., Frankfurt am Main, und vom Referat Film/AV-Medien der Zentralstelle Medien der Deutschen Bischofskonferenz, Bonn. Ort: Wechselnd; Zeit: Letzte Woche im Oktober.

b) Der Vertrieb vervollständigt Werbemaßnahmen von dem Augenblick an, wo ein Kunde sich für den Kauf eines AV-Mediums entschließt. Korrekte Rechnungsstellung und prompte Lieferung sind aus der Sicht des Käufers besonders wichtig; betriebsintern gewinnen Rationalisierungsmaßnahmen bezüglich Kopienlagerung, Verpackung und Versand sowie 
Inkassofragen und Kundenführung an Bedeutung. Nicht unwichtig sind in dem Zusammenhang auch Uberlegungen hinsichtlich Sonderangeboten, Rabatten und Rückgaberechten von (Ansichts-) Kopien. Vertriebsformen für AV-Medien sind unter anderem:

- Direktverkauf/Direktbelieferung (Versandhandel)

- Verkauf und Belieferung über den Lehrmittel-Fachhandel

- Verkauf und Belieferung über Sortimenter und den Buchhandel

- Verkauf und Belieferung uiber Verlags- und Phonoringe

Zu wünschen ist, daß sich die Kirchen - langfristig gesehen - allmählich eine eigene Infrastruktur zur A bnahme von Medienerzeugnissen aller Art schaffen (einschließlich Buch und Presse), wie sie im staatlich-kommunalen Bereich durch die Bildstellenorganisation dem lnstitut für Film und Bild in Wissenschaft und Unterricht FWU mit rund 450 Verleihstellen zur Verfügung steht. Allein von katholischer Seite bieten sich hierfür beispielsweise 774 Dekanate an - neben den dezentralen Materialstellen und 22 AV-Medienzentralen der Bistümer.

c) Die Qualität der AV-Produktionen - sowohl unter technischen wie unter didaktischen und inhaltlichen Aspekten - wird mehr und mehr zum entscheidenden Kriterium für die Beschaffung von AV-Medien: Die äußere Aufmachung, formale Gestaltung, didakt ische Verwendbarkeit, inhaltliche Relevanz und klare Zielgruppenausrichtung zählen - um so mehr, als aufgrund geschmolzener Etats faktisch nicht mehr alle Neuerscheinungen von Individualkunden, Bildungseinrichtungen oder AV-Medienzentralen erworben werden können. Natürlich spielt für den Verkauf nach wie vor auch der Preis eine Rolle! Leider sind AV-Medien in den letzten Jahren teuer geworden, für den Privatkunden zu teuer! AV-Verlage beklagen jedenfalls den Verlust an Einzelkunden: Erzieher, Lehrer, Referenten im außerschulischen Bildungsbereich, Pastoralassistenten und Pfarrer kaufen „privat", aus der eigenen Tasche, kaum noch audiovisuelle Lehr- und Lernmittel an. Vielleicht könnten mit einer entsprechenden Preisgestaltung, die sich etwa am Buchmarkt orientiert, die Einzelkunden wieder zurückgewonnen werden: Mit einfachen, weniger umfangreichen, nicht so kostenaufwendigen AV-Produktionen (Kleinmedien).

Statistik: Meistverkaufte Film-Titel

Institution/Film-Titel

verk. Kopien

Freigabe-Jahr

Institut für Film und Bild

FWU (Stand: 31.12.1980)

Quick - Das Eichhörnchen

(32 1712)

$1 / 54$

Schwangerschaft und Geburt

(32 0836)

$2.259 \quad 1 / 66$

Tiere im Winter (32 2308)

1.502

$1 / 72$

Favela - Ein Leben in Armut

(32 2350)

$1.115 \quad 1 / 72$

Der Muezzin ruft (32 0549)

1.100

$1 / 60$

\section{Referat Religion und Ethik}

im FWU (Stand: 31.12.1982)

Dir muß er ja nicht gefallen

(32 3I25)

Du lügst ja (32 300l) 
Was ist los mit Tommy?

(32 3000)

463

427

Einfach abtreiben? (32 3198)

Rückkehr (Gleichnis-Film,

32 3173)

317
$2 / 79$

$3 / 81$

$8 / 80$

rund 250

rund 250

rund 200

rund 200

rund 200

Angesichts einer wachsenden supranationalen Verflechtung der Bildungsbereiche und Bildungsbemühungen - gerade auch im kirchlichen Bereich - sowie einer sich abzeichnenden enormen Ausweitung von Sendemöglichkeiten im Zusammenhang mit der Entwicklung neuer Medien (Kabel- und Satellitenfernsehen, Video- und Bildplattensysteme), gewinnen Lizenzverkäufe (Veräußerung von Unterverwertungsrechten) ein besonderes Gewicht. Bei Verhandlungen mit ausländischen Interessenten spielen Fragen der Übertragbarkeit, der Inkulturation von AV-Medien im Abnehmerland eine große Rolle.

d) In der Bundesrepublik Deutschland gibt es derzeit rund 50 AV-Medien-Verlage und kirchliche oder kirchennahe Organisationen und Einrichtungen, die kontinuierlich Kleinmedien und Filme (audiovisuelle Gruppenmedien, Unterrichtsmedien, Bildungsmittel) für religiöse Erziehung, kirchliche Bildungsarbeit und Gemeindepastoral planen, herstellen und/oder vertreiben. ${ }^{15}$ Dicse Hersteller mit unterschiedlichem Produktionsprogramm und Stellenwert auf dem Markt leisten insgesamt einen nicht zu unterschätzenden Beitrag zur Verbreitung christlicher Wertvorstellungen und Informationen über den Glauben in unserer Gesellschaft. Tiefgreifende Veränderungen der letzten Jahre - zum Beispiel eine wachsende Zahl von Anbietern an AV-Medien, Steigerung der Produktionskosten, verbunden mit einer Verringerung des Absatzes, allmähliche Sättigung des Marktes (Ưberangebot?), Kürzung der Beschaffungsmittel bei Schulen, außerschulischen Bildungseinricht ungen und Pfarreien, hervorgerufen durch die allgemeine wirtschaftliche Rczession, Entwicklung neuer AV-Technologien, deren Nutzung durch die AV-Verlage zunächst hohe Investitionen erfordert, Tendenzen zur Bildung immer größerer Produktionsgesellschaften im AV-Bereich mit dem Ziel, die ökonomische Basis zu verbessern und den Einfluß auf dem Markt zu erhalten und nach Möglichkeit zu erweitern - all das hat dazu geführt, daß sich eine Reihe von AV-Verlagen und Filmgesellschaften derzeit in einer schwierigen wirtschaftlichen Phase befinden, die zum Teil existenzbedrohende Formen angenommen hat.

Ich halte die Zeit für die Einrichtung einer kirchlichen (katholischen) AV-Medienankaufsund -verkaufsstelle, die auf internationaler Ebene tätig ist, für überreif. Weltweit gesehen gibt es meines Erachtens kein Unternehmen und keine Organisation, die für die Verbreitung von AV-Medien eine ähnlich günstige Infrastruktur im Erziehungs- und Bildungsbereich aufzuweisen hätte: Sie besitzt ein weltverzweigtes Netz von Kontaktstellen und Einrichtungen, ähnliche Erwartungen und Bemühungen, relativ homogene Wertsysteme und Zielvorstellungen und einen internationalen Zusammenhalt! Aufgabe einer solchen internationalen kirchlichen Vertriebsstelle für audiovisuelle Medien müßte es sein, den Markt zu beobachten, den Bedarf zu erheben, Lizenzen der besten Produktionen zu erwerben und 
Kopien zu verbreiten! Gewisse Voraussetzungen hierfür bietet zweifellos die Arbeitsstelle für audiovisuelle Gruppenmedien in Brüssel/ Belgien, die von der Internationalen Katholischen Organisation für Hörfunk und Fernsehen UNDA sowie von der Internationalen Katholischen Filmorganisation OCIC gemeinsam getragen wird.

\section{Feedback und Evaluation}

Das immer noch schwächste Glied in der gesamten Medienarbeit bilden Feedback und gezielte Evaluation aufgrund von Rückmeldungen. Die meisten Produzenten wissen derzeit nicht, wie ihre AV-Medien bei dem von ihnen angezielten Publikum ankommen, welche Wirkungen sie in Unterricht, Bildung und Pastoral haben. Eine systematische Medienwirkungsforschung, speziell im kirchlichen Bereich, steckt noch in den Kinderschuhen. Exakte Untersuchungen über Funktion und Wirkung audiovisueller Medien im Zusammenhang religionsunterrichtlicher Praxis liegen meines Wissens bisher nicht vor. Aktuelle wie vor allem grundsätzliche U'berlegungen lassen solche Forschungsvorhaben jedoch erforderlich erscheinen. Dabei sollen Daten gesammelt und Faktoren bestimmt werden, die Folgerungen fur die fachdidaktische Theorie, für Gestaltung und Verwendung von AV-Medien und für weitergehende Untersuchungen auf dem Gebiet zulassen. Langfristig können sie dazu führen, die Effektivität religionsunterrichtlichen Handelns zu fördern, sich liber Verfahren, Inhalte und Ziele genauer zu verständigen und das Gespräch über religiöse Erziehung und AV-Medien sachlich zu führen. In dreifacher Hinsicht müßten AV-Medien in ihrer Leistungsfähigkeit erforscht werden: Als Lernhilfe für Schüler, als Lehrmittel für Lehrer und als Medien zur Vermittlung religiöser Praxis und Lebensformen, von Glaubensinformationen und christlichen Wertvorstellungen.

Defiziterfahrungen dieser Art haben im Referat Religion und Ethik des Instituts für Film und Bild FWU im Sommer 1979 zur Gründung eines „Testrings AV-Medien Religion“ geführt. Aufgabe des FWU-Testrings ist es, neue AV-Medien in konkreten „Verwendungssituationen“ zu erproben und darüber Erfahrungsberichte zusammenzustellen. Die Ergebnisse werden den Herstellern als Feed back-Material in vollem Umfang zur Verfügung gestellt; sie dienen darüber hinaus der Erstellung pädagogischer Medienbegleitmaterialien, der Abfassung von Rezensionen und Praxisberichten für entsprechende Fach- bzw. Zielgruppen-Zeitschriften. Auf diese Weise werden Interessenten auf neue AV-Produktionen aufmerksam gemacht und die Lücke zwischen Medienproduzenten einerseits und Mediennutzern andererseits wird geschlossen. Alle wichtigen AV-Verlage, einige Zeitschriften und insgesamt rund 50 Lehrer, Erzieher und Bildungsreferenten aus dem gesamten Bundesgebiet beteiligen sich am FWU-Testring AV-Medien Religion. Pro Jahr werden etwa 40 Neuproduktionen jeder Art und Gattung, im allgemeinen in zwei Einsätzen, in der Unterrichts- und Bildungspraxis getestet.

Eine interessante - wenn auch in Einzelfällen nicht immer unumstrittene Funktion - üben in dem Zusammenhang die sogenannten „Auswahlgremien“ der AV-Medienzentralen aus. Ihnen gehören Vertreter aller wichtigen kirchlichen Bildungsbereiche an. Sie haben die Aufgabe, Neuproduktionen zu sichten und über den Ankauf je nach Bedarfslage und vorhandenen Finanzmitteln zu entscheiden. Dabei üben sie eine Selektionsfunktion aus, die sich auf die Bewertungen der gesichteten AV-Medien stützt. Ein Erfahrungsaustausch dieser Auswahlgremien über die Kriterien ihrer Bewertungspraxis wäre zweifellos aufschlußreich!

Ein Erfahrungsseminar, bei dem alle Beteiligten an der Medienplanung und -produktion, am Medienvertrieb, -einsatz, -kritik und an der Mediennutzung über ihre Probleme praktischer und theoretischer Art berichten und Meinungen austauschen, wäre zweifellos sinnvoll. Leider hat es bislang solche Zusammenkünfte von Autoren, Redakteuren, Regisseuren, Kameraleuten, Kritikern, Lehrern, Erziehern, Bildungsreferenten, Geistlichen, Produzenten, 
Medienverleihern und Wissenschaftlern zu selten gegeben! Vor Jahren wurde in München eine entsprechende Tagung über „Biblische Geschichten“ durchgeführt, an der sowohl Exegeten wie Katecheten und Fachleute aus dem AV-Bereich teilgenommen haben. Im Ergebnis hat das Seminar für spezifische Probleme sensibilisiert und zu einer Reihe von Verbesserungen in der Zusammenarbeit zwischen „Machern" aus verschiedenen Arbeits- und Fachbereichen geführt. Ein ähnlicher Erfahrungsaustausch wurde im Zusammenhang mit der Planung und Produktion einer Filmreihe für die Sakramentenpastoral durchgeführt.

Viel bleibt noch zu tun, um Aufschluß zu erhalten über unterschiedliche Formen des Medieneinsatzes in pädagogischen und didaktischen Zusammenhängen sowie über ihre Wirkung auf die Rezipienten. Eine Beteiligung von Fachwissenschaftlern aus den theologischen Fakultäten unserer Universitäten, aus den theologischen Hochschulen und religionspädagogischen Instituten erscheint immer dringlicher und unausweichlich.

\section{Zum Verhältnis von Theologie und Audiovision}

In einer Resolution, die von Teilnehmern des „Ersten Weltkongresses über audiovisuelle Medien und Verkündigung“ (München, 6.-10.11.1977) verabschiedet worden ist, heißt es: „Der Kongreß bekräftigt die Notwendigkeit wissenschaftlicher Forschung, ohne die es keinen ernsten Fortschritt in Herstellung und Gebrauch audiovisueller Medien geben kann“. ${ }^{16}$ Auch in der Pastoralinstruktion über die Instrumente der sozialen Kommunikation „Communio et Progressio“ vom 23. Mai 1971 wird gefordert: „Alle formalen und inhaltlichen Probleme der sozialen Kommunikation sollen auch ihren Ort in den Lehrplänen der Theologie finden, wo immer sie den Lehrstoff berühren, vor allem in der Moral- und Pastoraltheologie. Die Grundzüge dieser Fragen sollen darüber hinaus in die katechetischen Lehrbücher aufgenommen werden" 17 .

Die Theologie als Wissenschaft kann meines Erachtens vor allem auf drei Ebenen einen Beitrag zur Bewältigung anstehender Probleme im AV-Bereich leisten:

a) Wissenschaftliche Forschung. Hier zeichnen sich vier größere Arbeitsfelder ab:

- Geschichtliche Untersuchungen, zum Beispiel zur Problematik der Filmbewertung aus kirchlicher Sicht oder zur Geschichte der internationalen katholischen Medienorganisationen UCIP (Union Catholique Internationale de la Presse), UNDA (Internationale Katholische Rundfunk-Organisation) und $\mathrm{OCIC}$ (Office Catholique International du Cinema);

- Empirische Erhebungen, beispielsweise über Nutzung und Auswirkung des Schulfernsehens im Religionsunterricht oder eine vergleichende Studie zum kirchengeschichtlichen Unterricht mit und ohne AV-Medien;

- Theoretische Abhandlungen, zum Beispiel über die Visualisierung biblischer Gleichnisse (in Filmen) oder über die Frage, wie die Kirche ihren Bedarf an Forschung im Bereich der AV-Medien am besten organisieren und daraus resultierende Ergebnisse vorteilhaft nutzen kann;

- Systematische Sammlung und Auswertung von Informationen, zum Beispiel durch den Aufbau einer Datenbank/Dokumentation für AV-Medien in religiöser Erziehung und kirchlicher Bildungsarbeit beziehungsweise durch die Erstellung eines Verzeichnisses von wissenschaftlichen Arbeiten, Persönlichkeiten, Organisationen und Institutionen, die sich auf nationaler und internationaler Ebene mit AV-Fragen beschäftigen.

b) Lehre (Aus- und Fortbildung). Hand in Hand mit Produktion und Verbesserung des Medienverleihs muß eine mediendidaktische Ausbildung und Fortbildung gehen. Erst die Kenntnis kommunikativer Abläufe und die Beachtung mediendidaktischer Gesichtspunkte 
ermöglichen eine effektive Verwendung dieser Lehr- und Lernmaterialien. Zwei Bereiche sind dabei vor allem zu beachten:

- Medienpädagogische und mediendidaktische Ausbildungs- und Fortbildungsprogramme für unterschiedliche Zielgruppen im kirchlichen Bereich wären zu entwickeln, zum Beispiel für Kindergärtnerinnen, Religionslehrer, Referenten der theologischen Erwachsenenbildung;

- Zu sorgen ist aber auch für die Ausbildung von Spezialisten: Drehbuchautoren, Regisseure, Redakteure, Fernsehmoderatoren, Filmjournalisten oder ähnliche. Wir müssen jungen Menschen Mut machen - und sie unter Umständen auch finanziell unterstützen damit sie Berufe im Bereich der Medienproduktion und Medienkritik ergreifen.

c) Die Mitarbeit eines Theologen an konkreten AV-Projekten könnte als Autor oder als Fachberater erfolgen. Eine fruchtbare Mitwirkung ergäbe sich bei theologischen Kursen im Medienverbund, wie es etwa bei der Fernsehserie „Warum Christen glauben“ der Fall gewesen ist ${ }^{18}$; besonders wichtig wäre eine Beteiligung auch bei der Entwicklung von medienpädagogischen und mediendidaktischen Curricula. In dem Zusammenhang möchte ich wenigstens auf je eine nationale und internationale Einrichtung hinweisen, die im Bereich von Lehre und Forschung Beachtliches leisten:

1976 wurde an der Münchner Jesuitenhochschule für Philosophie das „Institut für Kommunikation und Medien“ gegründet. Da gibt es jederzeit eine ständige Studiengruppe „Bildschirmtext“, eine Projektgruppe „Kabelfernsehen“ und eine Planungsgruppe „Bildplatte“. Das Thema „Neue Medien" wird nicht nur theoretisch erörtert, sondern die Mitarbeiter versuchen bei der konkreten Ausgestaltung dieser neuen Kommunikationsmöglichkeiten einen Beitrag zu leisten. Eine weitere Arbeitsgruppe beschäftigt sich mit der „Wertvermittlung durch Fernsehen“ und mit einer Untersuchung über „Religion und Ethik in der Bild-Zeitung". Nicht alle möglichen Aspekte der modernen Kommunikation werden untersucht, sondern vor allem solche, die der Kirche wichtig sein müßten. Es geht also vor allem um ethische Gesichtspunkte im Rahmen von Kommunikation und Medien, um Inhalte und Formen religiöser Mitteilungen in den öffentlichen Medien und schließlich um das Kommunikationsverhalten von kirchlichen Gruppen und der Kirche insgesamt. Anschrift: Institut für Kommunikation und Medien, Kaulbachstr. 3la, 8000 München 22.

Das „Centre for the Study of Communication and Culture CSCC" versteht sich als internationales kirchliches Service-Unternehmen im Bereich von Kommunikation/AV-Medien und Forschung. Das Institut selbst führt nur in Ausnahmefällen eigene Forschungsvorhaben durch, es sieht seine Aufgaben vor allem darin, Untersuchungen anzuregen und bei deren Durchführung Unterstützung zu leisten. Dies geschieht durch eine systematische Erhebung und Feststellung des Bedarfs, durch die Koordination von Forschungsvorhaben sowie durch die Zusammenführung entsprechender Fachwissenschaftler. Hierfür steht ein Dokumentationszentrum zur Verfügung und ebenso der Informationsdienst "Communication Research Trends"; von Zeit zu Zeit werden auch internationale Seminare für Forscher durchgeführt. Anschrift: Centre for the Study of Communication and Culture, 221 Goldhurst Terrace, London NW6 3EP, England.

\section{Anmerkungen}

15 Vgl. A. Täubl: Zur Situation kirchlich-orientierter AV-Verlage in der Bundesrepublik Deutschland, in CS, 14. Jg. (1984), S. ff.

16 Audiovisual and Evangelisation. World Congress. Acta Congressus AV-EV. IV Recommendations, S. 9, Vatikan: Sekretariat SM-OCIC, 1977.

17 Communio et Progressio, Nr. 108

$18 \mathrm{Vgl.:}$ Warum Christen glauben. 13-teilige Fernsehreihe, Katholische Landesarbeitsgemeinschaft für Erwachsenenbildung Rheinland-Pfalz (Hrsg.), Trier: Spee-Verlag, 1979. 


\section{SUMMARY}

In the second part of the contribution the preparation and production of short films is treated like the contacts to authors, the consultation with specialists, development of production outline like expose, treatment, scriptbook, the contracts and execution of the productions, as well as the accompanying material. Also the risk of productions is discussed. A special chapter is devoted to advertising, distribution and sales. For this the technical, didactic and content quality of the productions are becoming more and more decisive. Feed back and evaluation are also quite important even though they are often the most weak of the whole media work.

The last chapter points to three areas important for solving problems in audio-visual work: scientific research, training and formation of experts and multiplicators, and the cooperation of theologians on concrete projects as authors and consultants.

\section{RESUMEN}

La segunda parte del artî de Täubl se refiere a la preparación y producción de films cortos, los contactos con los autores, las consultas con los especialistas, el desarrollo de las principales lineas de la producción tales como la exposición, el tratamiento, el libreto, los contratos y la ejecución de las producciones asî como del material de acompan̂amiento. También se presentan los riesgos de la producción. Un capîtulo especial ha sido dedicado a la publicidad, distribución y ventas. Por esto la calidad técnica, didáctica y de contenido de las producciones se vuelve cada vez más decisiva.

La retroalimentación y la evaluación son también una parte importante, más aún cuando se piensa que muy a menudo son la parte más débil del conjunto del trabajo en los media. El último capîtulo destaca tres importantes áreas para resolver problemas en el trabajo audio-visual: investigación cientîfica, capacitación y formación de expertos y multiplicadores y la cooperación de teólogos en la producción, tanto como autores y consultantes para la elaboración de proyectos concretos.

\section{RESUME}

L'auteur commente dans cette deuxiéme partie de sa contribution des problémes concrets de la préparation et production de films de court métrage, comme les contacts avec les auteurs, les consultations avec des spécialistes, le développement de lexposé, le traitement, le script, les contrats et l'exécution des productions, jusqú au matérial pédagogique d'accompagnement. Aussi les risques de production sont mentionnés. Un chapitre est dévoué à la publicité, la distribution et la vente des média $A V$, avec la remarque que la qualité des productions, sous les aspects techniques, didactiques et du contenu, devient de plus en plus un critére décisif pour leur achat. L'auteur ajoute un paragraphe sur le feed-back et l'évaluation, malgré qú ils représentent toujours les points faibles dans tout le travail des média.

Dans la dernière partie, l'attention est attirée sur trois champs de travail, dans lesquels la théologie pourrait faire une contribution à la solution de problèmes existants dans le domaine de l'audiovisuel: La recherche scientifique, formation des experts de média et la coopération de théologiens comme auteurs et conseillers. 